\title{
Cold Atmospheric Plasma (CAP) for the Treatment of Actinic Keratosis and Skin Field Cancerization: Clinical and High-Frequency Ultrasound Evaluation
}

\author{
Mariachiara Arisi (D) - Simone Soglia · Edoardo Guasco Pisani • \\ Anna Venturuzzo • Alessandra Gelmetti · Cesare Tomasi • \\ Cristina Zane $\cdot$ Mariateresa Rossi $\cdot$ Luisa Lorenzi $\cdot$ Piergiacomo Calzavara-Pinton
}

Received: January 27, 2021 / Accepted: March 6, 2021 / Published online: March 18, 2021

(C) The Author(s) 2021

\section{ABSTRACT}

Introduction: Cold atmospheric plasma (CAP) has been clinically demonstrated to be an effective treatment for actinic keratosis (AK) in a number of case series. In this study, we evaluated the efficacy of CAP in the treatment of multiple AKs and assessed morphological changes induced on the skin field of cancerization both clinically and by high-frequency ultrasound (HFUS).

Methods: Patients with multiple grade I-II AKs of the scalp and/or face who were resistant or intolerant to conventional field-directed treat-

M. Arisi $(\bowtie)$. S. Soglia · E. Guasco Pisani .

A. Venturuzzo - A. Gelmetti - C. Zane - M. Rossi ·

P. Calzavara-Pinton

Dermatology Department, ASST Spedali Civili di Brescia, University of Brescia, P.le Spedali Civili 1, 25123 Brescia, Italy

e-mail: mariachiara.arisi@gmail.com

C. Tomasi

Department of Clinical and Experimental Sciences, University of Brescia, Brescia, Italy

L. Lorenzi

Department of Pathology, ASST Spedali Civili di

Brescia, University of Brescia, Brescia, Italy ments were enrolled. CAP treatments were performed using a microwave-driven argon plasma jet. At baseline and 3 months after the last CAP session, performance indexes were determined using three-dimensional digital pictures and HFUS investigations were performed on a representative Olsen grade II AK and a small spot of clinically unaffected skin within the test area.

Results: Twelve patients were enrolled in the study. All clinical variables showed a statistically significant reduction after CAP. HFUS evaluation revealed that the total, epidermal and dermal thicknesses of the target AKs had not changed with treatment. CAP therapy significantly increased dermal density in both the target $\mathrm{AK}$ and the surrounding photodamaged skin and signficantly decreased the thickness of the subepidermal low-echogenic band in the perilesional skin, which is an ultrasound sign of photodamage.

Conclusions: Cold atmospheric plasma was found to be an effective treatment for patients with multiple AKs. CAP was not followed by skin atrophy. HFUS examiniation showed the CAP improved features of chronic photodamage of the dermis of the skin underlying and surrounding the AK spots.

Keywords: Cold atmospheric plasma; Actinic keratosis; Skin field cancerization; Highfrequency ultrasound 


\section{Key Summary Points}

Treatment of multiple actinic keratoses can be challenging due to poor treatment efficacy or poor patient compliance.

Cold atmospheric plasma is a novel therapeutical approach for the treatment of multiple actinic keratoses.

\section{DIGITAL FEATURES}

This article is published with digital features, including a summary slide, to facilitate understanding of the article. To view digital features for this article go to https://doi.org/10.6084/ m9.figshare.14171132.

\section{INTRODUCTION}

Plasma, considered to be the fourth state of matter, is generated by ionizing neutral gas molecules through the use of energy in the form of heat or electromagnetic fields [1]. Plasma consists of charged ions, reactive oxygen species (ROS), reactive nitrogen species and energetic photons (ultraviolet [UV]) and excited molecules, and it generates transient electric fields [2]. There are two types of plasma: thermal and nonthermal plasmas (NTP); both are defined by the temperature of electrons compared to the temperature of the other particles [3]. Thermal plasmas are commonly used in endoscopic surgery [4], while the development of devices generating cold atmospheric plasmas (CAP) has allowed the use of CAP in several applications, without the potential of harming the treated tissue. The biological effects of NTP on tissues appear to be dose dependent [5], with low doses inducing inactivation of bacteria and cell proliferation $[6,7]$ and high doses being able to induce apoptosis of the targeted cells [8]. Based on these assumptions, CAP has been used in several dermatological applications in recent years, such as the disinfection and healing of wounds and treatment of infective and inflammatory skin diseases [9]. However, the application field that has recently attracted increasing interest is oncology [10]. A number of studies have evaluated the application of plasma for the treatment of skin cancer in vitro $[11,12]$ and in vivo in murine models $[11,13]$. The literature also contains a few examples of CAP therapy for the treatment of skin cancers $[14,15]$ and precancerous lesions $[16,17]$ in human patients.

The presence of CAP-originated reactive species causes a noticeable increase in the level of intracellular ROS; this increase cannot be countered by the intracellular antioxidant systems and therefore causes further serious DNA double-strand breaks. The result is the occurrence of cell-cycle arrests and apoptosis based on disruption of the mitochondrion pathway or tumour necrosis factor receptor pathway [18].

The CAP-induced anti-cancer effect is selective: CAP-originated reactive species diffuse significantly faster into cancer cells than into healthy cells due to a higher expression of aquaporins on the cytoplasmic membrane of the former [18]. Furthermore, the tumour selectivity and apoptotic effects of CAP are especially high in p53-mutated cancer cells [19] and during the DNA replication phase, which in a large percentage of cancer cells is a very prominent phase [20]. Actinic keratoses (AKs) and the underlying UV-induced field of cancerization are the most common precursors of cutaneous squamous cell carcinoma (cSCC) [21]. As a result of their well-documented potential to evolve to invasive skin tumours, several strategies have been developed for their treatment, both physical and pharmaceutical [22]. However, despite the large number of available treatment options, management of AKs and the skin field of cancerization is sometimes challenging due to undesirable treatment-induced local side effects and the consequent poor tolerance and adherence of the patient to treatment.

In this context, the effectiveness of CAP in the treatment of AK has been demonstrated in some case series $[17,23]$. We present here our experience with the application of CAP for the treatment of resistant AKs of the face and/or scalp. The efficacy of CAP was investigated both 
clinically and by high-frequency ultrasound (HFUS) examination.

\section{METHODS}

\section{Patients}

Adult patients (phototype I-IV) with multiple $(\geq 5)$ grade I-II [24] AKs of the scalp and/or face that were resistant or intolerant to at least three previous conventional field-directed treatments were enrolled in the study. The patients were attending the Dermatology Department of Brescia University from April 2019 to October 2019. Exclusion criteria were: concomitant clinically significant unstable medical conditions; active systemic infectious diseases; immunosuppression; chemical dependency or alcoholism; current participation in another clinical study; invasive tumours within the area of treatment; use of photosensitizing drugs; pregnancy or lactation; any other dermatological disease in the treatment area or within a distance of $3 \mathrm{~cm}$; prior physical or topical treatment for AKs within a period of 6 months; and likelihood of poor compliance.

The study was approved by the Local Ethics Committee (Protocol Number 4195) and was conducted in accordance with the Declaration of Helsinki. All patients were given verbal and written information on the nature of the study, and all signed an informed consent before enrolment. All patients provided written informed consent for the publication of their cases and images.

\section{Treatment Procedure}

The CAP treatments were performed with a CEcertified microwave-driven argon plasma jet (Adtec SteriPlas; Adtec Plasma Technology, Adtec Europe, Hunslow, UK; Fig. 1) according to the following protocol: 120 -s sessions, twice a week until resolution and treatment for a maximum of 7 weeks [17]. The device consists of a torch operated at microwave excitation and the production of an argon plasma [25]. The emission spectrum peak in the UVA and UVB range

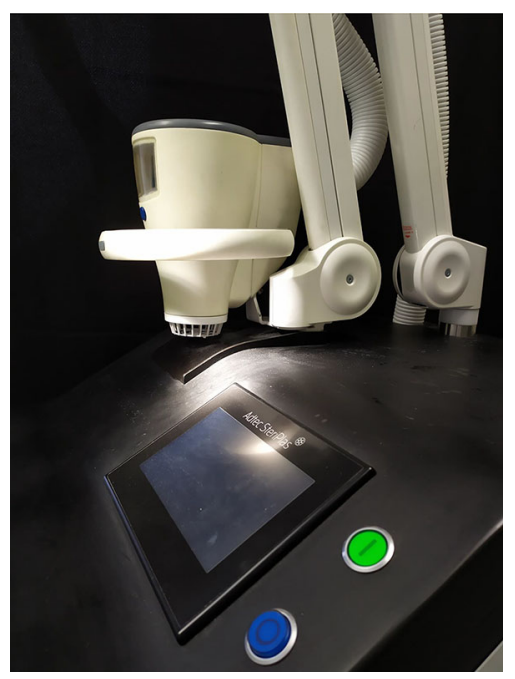

Fig. 1 Equipment used to generate the cold atmospheric plasma (CAP) jet used during the treatment of actinic keratoses (AKs)

was 308 and $336 \mathrm{~nm}$ respectively, measured using a StellarNet Silver Nova spectrometer (StellarNet Inc. Tampa, FL, USA).

In all patients, the treatment procedure was performed under standard conditions, with the environmental temperature and humidity ranging between $20^{\circ} \mathrm{C}$ and $23^{\circ} \mathrm{C}$ and between 40 and $60 \%$, respectively, and the patient remaining in a lying position for at least $10 \mathrm{~min}$ before treatment initiation. Taking into consideration that the diameter of the plasma handle is $227.4 \mathrm{~mm}$ and a single application of plasma consequently encloses a field of $12 \mathrm{~cm}^{2}$, in each session multiple applications of plasma were needed to treat the entirety of the affected areas. The device used is a "spot style" and designed to fixate a few millimetres (approx. 5 $\mathrm{mm}$ ) over the lesion to be treated and to stay in place during the irradiation period. All patients were evaluated at baseline (T0) and 3 months (90 days) after the last CAP session (T1). At each time point, three performance indexes were determined: (1) the number of lesions were defined after visual inspection and dermoscopical examination (Vidix 4.0; Medici Medical, Modena, Italy) by two investigators (MA, AG); (2) the cumulative area of the AKs in the sample $[26,27]$ were calculated during the analysis (Vectra Viewer® software; Canfield, Fairfield, 
NJ, USA) of three-dimensional (3D) digital pictures (Vectra H1 ${ }^{\circledR}$ camera; Canfield); (3) and the actinic keratosis area and severity index (AKASI) was calculated [27, 28].

In addition, we selected a representative Olsen grade II AK and a small spot of clinically unaffected skin within the test area for HFUS investigations. We used a 50-MHz HFUS apparatus (DUB ${ }^{\circledR}$ Skin Scanner; Taberna Pro Medicum, Lüneburg, Germany) that has a $4-\mathrm{mm}$ useful depth of signal penetration and a $40-\mathrm{dB}$ gain under standard conditions $\left(20-23^{\circ} \mathrm{C}\right.$ environmental temperature with patient lying down for at least $10 \mathrm{~min}$ ). To limit measurement errors, three measurements were carried out by the same operator in the same area of skin, and the average of the values was calculated. The main HFUS features of photodamage or hyperkeratosis were total, epidermal and dermal thicknesses $(\mu \mathrm{m})$, subepidermal lowechogenic band (SLEB) thickness $(\mu \mathrm{m})$ and lesional and SLEB echogenicity (expressed as percentage of dermal density), analysed with a specific image-analysis software. After treatment, HFUS investigations were repeated in the same skin spots that were identified on the basis of the digital pictures.

During each CAP session, patients rated pain and/or burning sensation on a standardized 1-10 visual analogue scale (VAS).

At T1 the overall cosmetic outcome of patients was graded by the same physicians (AM, AG) into four categories: excellent (no or mild redness or change in pigmentation), good (moderate redness or change in pigmentation), fair (slight-to-moderate scarring, atrophy or induration) and poor (extensive scarring, atrophy or induration) [29].

In only two patients were 4-mm incisional skin biopsies performed on a representative single AK in the treated skin area. The skin sample was analysed by staining with haematoxylin and eosin in order to highlight the possible persistence of $\mathrm{AK}$ after treatment.

\section{Statistical Analysis}

Statistical analysis was performed using SPSS software (v25.0; IBM SPSS, Armonk, NY, USA).
Categorical variables were summarized using percentages and continuous variables by calculating medians and range (minimum and maximum values). Normal distribution of collected data was analysed by the Kolmogorov-Smirnov test. The medians of continuous variables were compared using the Wilcoxon test. All results were considered statistically significant at $p \leq$ 0.05 .

\section{RESULTS}

Twelve patients were enrolled in the study and all completed the study. The main clinical details are described in Table 1.

All clinical variables (number and cumulative area of AKs, AKASI score) showed a

Table 1 Relevant clinical details of enrolled patients at baseline (T0)

\begin{tabular}{ll}
\hline Baseline patient clinical characteristics & Values \\
\hline Number of patients & 12 \\
Gender & $12(100 \%)$ \\
Male & $0(0 \%)$ \\
Female & 80 \\
Median age (years) & $51-89$ \\
Age range (years) & \\
Fitzpatrick's skin phototype & $2(16.7 \%)$ \\
I & $8(66.6 \%)$ \\
II & $2(16.7 \%)$ \\
III & $14(6-50)$ \\
Number of AKs at T0 (mean/range) & $5.5(2-26)$ \\
Grade I & $8(4-24)$ \\
Grade II & $10(83.3 \%)$ \\
Body site & \\
Scalp & $6(50 \%)$ \\
Face & \\
\hline
\end{tabular}

Values are presented as the number with the percentage in parenthesis, unless indicated otherwise $A K$ Actinic keratosis 
Table 2 Actinic keratosis area and severity index score and number and cumulative area of AKs at baseline and 3 months after end of cold atmospheric plasma treatment

\begin{tabular}{|c|c|c|c|c|c|}
\hline \multicolumn{2}{|l|}{ AKASI score } & \multicolumn{2}{|c|}{ Number of AKs } & \multicolumn{2}{|c|}{ Cumulative area of AKs $\left(\mathrm{cm}^{2}\right)$} \\
\hline Baseline & 90 days $\mathrm{EOT}$ & Baseline & 90 days $\mathrm{EOT}$ & Baseline & 90 days EOT \\
\hline $3.5(1.2-4.8)$ & $2.4(1.2-6)$ & $14(6-50)$ & $10(3-30)$ & $8.24(1.48-36.62)$ & $4.24(1.2-27.673$ \\
\hline \multicolumn{2}{|c|}{$p=0.05^{*}$} & \multicolumn{2}{|c|}{$p=0.01^{*}$} & \multicolumn{2}{|c|}{$p=0.01^{*}$} \\
\hline
\end{tabular}

Values in table are presented as the median with the range in parenthesis AKASI Actinic keratosis area and severity index score, EOT end of treatment

*Statistically significant difference at $p \leq 0.05$

statistically significant reduction after treatment (Table 2; Figs. 2, 3).

Improvement in the cumulative area of the AKs induced by treatment was between 11 and $83 \%$, and higher than $42 \%$ in half of the patients. Treatment was well tolerated and painless (VAS $=0$ ) in all patients. None of the patients experienced any adverse events during the treatment or at the 3-month follow-up.

HFUS analysis was performed in eight patients. At the HFUS evaluation, total, epidermal and dermal thicknesses of the target AKs had not changed after treatment. CAP significantly increased the dermal density of both the skin underlying the target $\mathrm{AK}$ and the surrounding photodamaged skin and significantly decreased SLEB thickness in the perilesional skin (Table 3; Fig. 4).

Cosmetic outcome was rated as excellent in ten $(83.3 \%)$ patients and good in two $(16.7 \%)$ patients.

Histological analysis of skin biopsies in two patients revealed the disappearance of the AK after plasma application (Fig. 5).

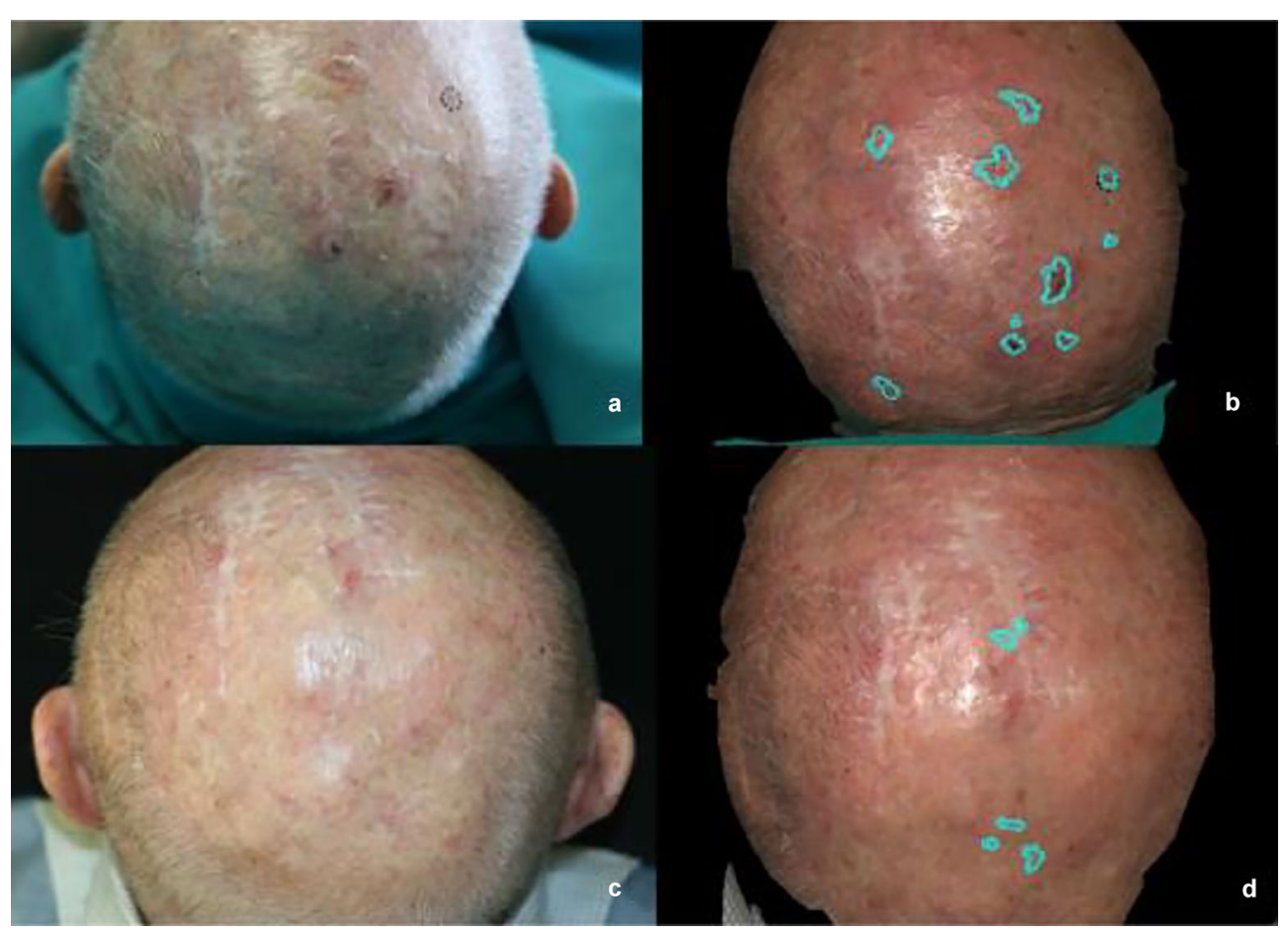

Fig. 2 Clinical evaluation of number and cumulative area (turquoise area) of AKs of the scalp by three-dimensional (3D) digital pictures. a, b Before CAP, $\mathbf{c}-\mathbf{d} 90$ days after end of CAP therapy 


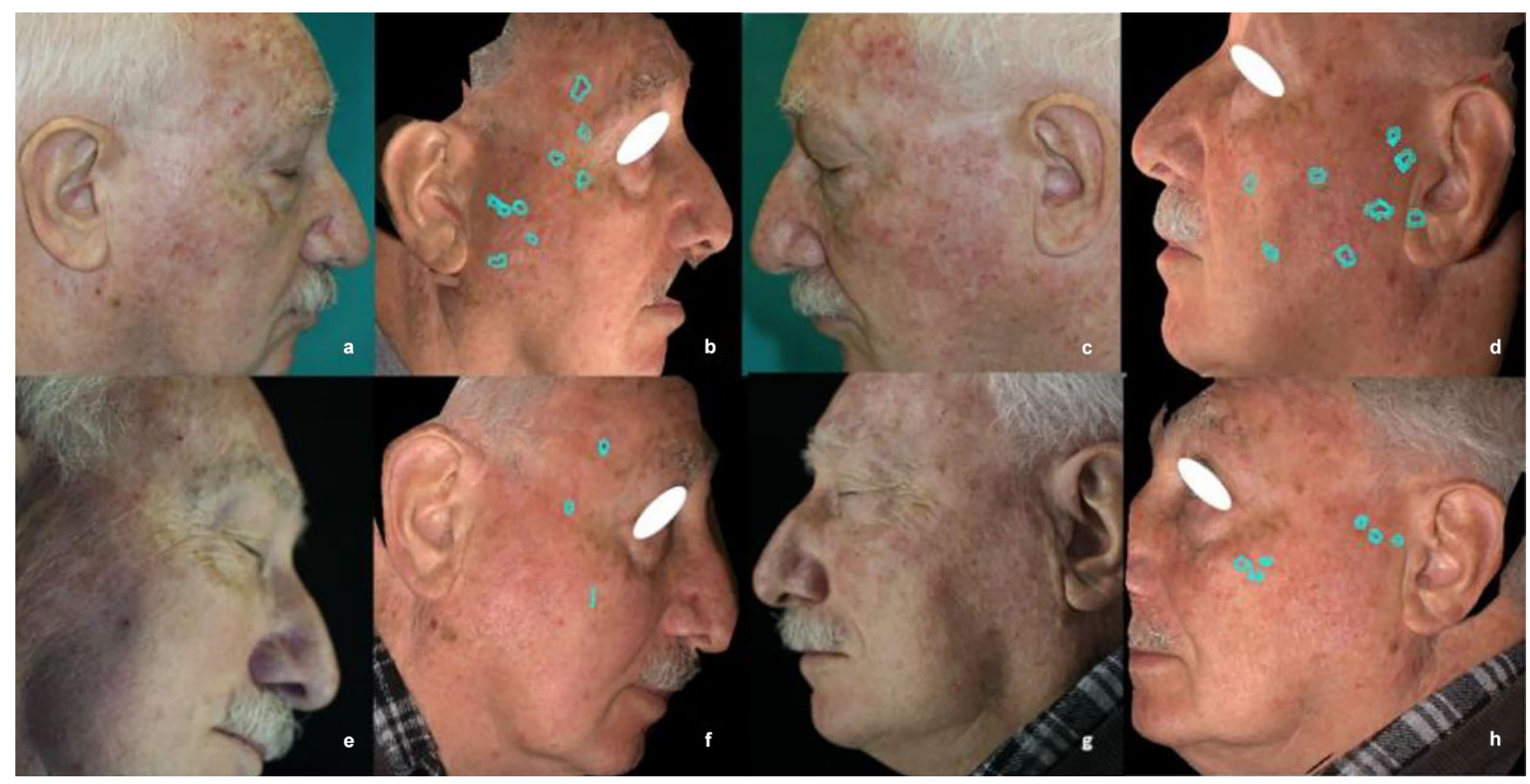

Fig. 3 Clinical evaluation of number and cumulative area (turquoise area) of AKs of the cheeks by 3D digital pictures. $\mathbf{a}-\mathbf{d}$ Before CAP, e-h 90 days after end of CAP therapy

Table 3 Changes in the target lesions and in the photodamaged surrounding skin at baseline and after cold atmospheric plasma treatment in eight selected patients according to high-frequency ultrasound examination

\begin{tabular}{|c|c|c|c|c|c|c|}
\hline $\begin{array}{l}\text { Time point } \\
\text { and } \\
\text { significance }\end{array}$ & $\begin{array}{l}\text { Target AK } \\
\text { total thickness } \\
(\mu \mathrm{m})\end{array}$ & $\begin{array}{l}\text { Target AK } \\
\text { epidermal } \\
\text { thickness }(\mu \mathrm{m})\end{array}$ & $\begin{array}{l}\text { Target AK } \\
\text { dermal } \\
\text { thickness }(\mu \mathrm{m})\end{array}$ & $\begin{array}{l}\text { Target AK } \\
\text { dermal } \\
\text { density (\%) }\end{array}$ & $\begin{array}{l}\text { Perilesional } \\
\text { SLEB density } \\
\text { (\%) }\end{array}$ & $\begin{array}{l}\text { Perilesional } \\
\text { SLEB } \\
\text { thickness }(\mu \mathrm{m})\end{array}$ \\
\hline T0 & $\begin{array}{l}937.5 \\
\quad(719-1391)\end{array}$ & $97.5(59-633)$ & $\begin{array}{l}730.5 \\
\quad(656-1133)\end{array}$ & $\begin{array}{l}10.7 \\
(2.2-17.4)\end{array}$ & $7.1(4.6-17.2)$ & $223(102-313)$ \\
\hline $\mathrm{T} 1$ & $\begin{array}{l}949.5 \\
\quad(816-1734)\end{array}$ & $91.5(82-976)$ & $762(727-1160)$ & $\begin{array}{l}12.9 \\
\quad(7.1-23.53)\end{array}$ & $\begin{array}{l}15.1 \\
\quad(6.4-40.2)\end{array}$ & $146.5(74-273)$ \\
\hline$p$ & 0.074 & 0.61 & 0.47 & $0.02^{*}$ & $0.02^{*}$ & $0.04^{*}$ \\
\hline
\end{tabular}

Values in table are presented as the median with the range in parenthesis

SLEB Subepidermal low-echogenic band, TO baseline, TI 3 months after the last cold atmospheric plasma (CAP) session ${ }^{*}$ Statistically significant difference between values at $\mathrm{T} 0$ and $\mathrm{T} 1$ at $p \leq 0.05$

\section{DISCUSSION}

In the present trial, CAP was found to be effective for the treatment of patients with multiple AKs of face and/or scalp. There was a statistically significant reduction in both the AKASI score and the number of AKs after CAP treatment in all 12 patients. The number of AKs is often considered to be the most reliable performance index [30, 31], although it can be influenced by the size of the lesions and by the degree of difficulty to accurate determine the number the AKs when the lesions are close to each other or partially confluent. Nonetheless, the results of the present study substantially confirm the outputs of two previous case series [16, 17]. In the first of these pilot studies to be published [16], 17 AKs were treated using a nonthermal 

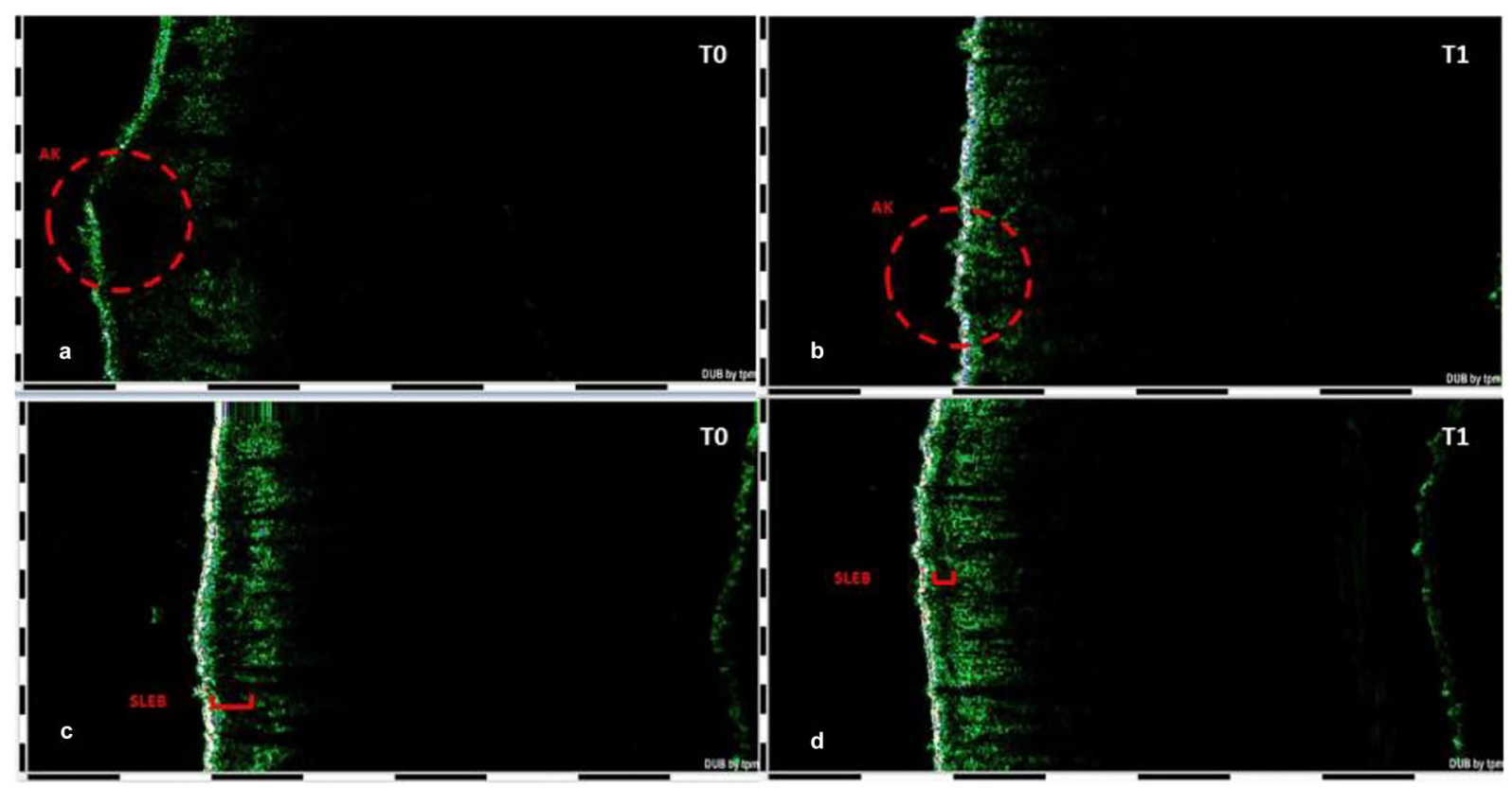

Fig. 4 High-frequency ultrasound study of a target AK lesion (inside red circle in $\mathbf{a}, \mathbf{b}$ ) and surrounding skin field of cancerization (in red in $\mathbf{c}, \mathbf{d}$ ) before and after CAP treatment in eight selected patients. TO Baseline, T1 3 months after the last CAP session. SLEB Subepidermal low-echogenic band

guided prediction of treatment efficacy in dermatology.

In our case studies, the CAP led to a percentage reduction in the area of the treated AKs compared to baseline of between 11 and 83\%, but more than $44 \%$ in half of our patients. These data were also influenced by the initial strongly compromised clinical condition of some patients who were candidates for CAP precisely because they had been resistant to many other skin field treatments.

In this study we also aimed to investigate the effect of CAP on the HFUS parameters of both lesional and chronically photodamaged skin surrounding the lesions. To date, the biological effects of CAP on AKs have not been evaluated through non-invasive imaging techniques, with the exception of a study involving optical coherence tomography (OCT), which showed a clearly demarcated dermoepidermal junction and no residual epidermal tissue left in 74\% lesions after CAP [32]. Notwithstanding, given the different resolution and penetration capabilities of OCT and HFUS, the results of our 


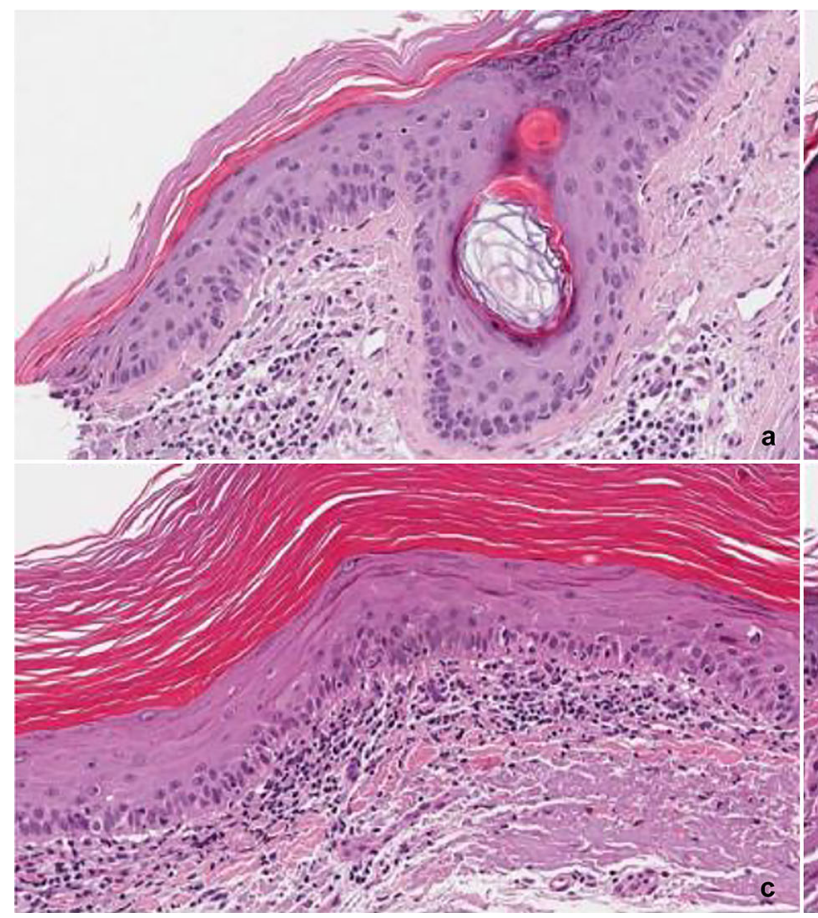

Fig. 5 Histological analysis of skin biopsies of two patients. a, b Patient 1. a Before CAP: at the left margin of the punch biopsy, hyperkeratosis with parakeratosis associated with atypia of basal keratinocytes and solar elastosis of the dermis are present, $\mathbf{b}$ after CAP: solar elastosis of the dermis remains evident while hyperkeratosis and keratinocyte atypia are absent. c, d Second patient. c

study are unlikely to be comparable to those obtained with OCT.

In our series, the total and dermal thickness of AK spots did not change after CAP treatment, suggesting that the clearance of lesions is not followed by skin atrophy. Furthermore, the epidermal thickness of the treated lesions remained almost constant, demonstrating that CAP treatment does not have a considerable effect on the hyperkeratosis. This result is in contrast to the results reported by Wirtz et al. [32] showing that CAP led to a reduction of AK thickness. However, we only compared our results with the clinical evaluation conducted by these authors [32] without the aid of noninvasive imaging methods. Moreover, the relevance of these data in clinical practice is limited to the currently corroborated assumption supporting that there is no correlation between

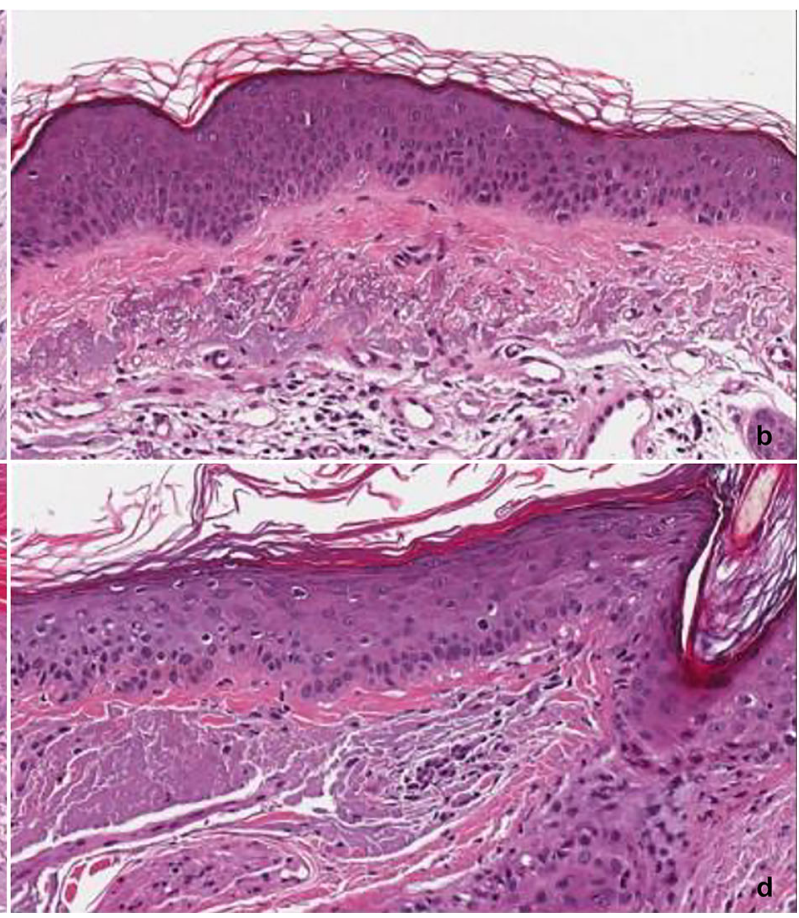

Before CAP: hyperkeratosis with orthokeratosis, mild atypia of keratinocytes and lichenoid inflammatory infiltrate in the papillary dermis associated with elastosis are present, $\mathbf{d}$ after CAP: there is no sign of hyperkeratosis and of the dermal inflammatory infiltrate in the biopsy

histological and Olsen clinical grading and the risk of progression of AKs to invasive SCC [33].

In our study we also investigated the perilesional cancerization field by ultrasound, as this field is also inevitably affected by CAP treatment.

The two main HFUS features of photodamage are dermal echogenicity, which correlates to dermal density, and the thickness of the SLEB [34]. CAP treatment significantly increased the dermal density underlying the AK spots and significantly decreased the SLEB thickness of the perilesional skin together with an increase of its density. The increase of the echogenicity of the upper and lower dermal layers and the reduction of the SLEB thickness with CAP treatment can be related to several concurrent biological effects, including fibroblast proliferation and collagen synthesis [35] and the removal of elastotic fibres by recruited immune cells [36]. 
In vivo and in vitro studies showed a significant induction of mRNA expression for transforming growth factor beta [37] after exposure to plasma, which regulates fibroblast migration and proliferation [38]. CAP induces $\alpha$-smooth muscle actin and COL1A1 (alpha-1 type I collagen) expression, providing extracellular matrix deposition and remodelling [35].

The new apposition of collagen fibres also supports the increase in dermal thickness seen in the ultrasound examination at $\mathrm{T} 1$ and the consequent increase in the total thickness.

The effect of plasma on the field of cancerization can be also explained by its ability to oxidate redox-sensitive cysteine and thiols in proteins, which can evoke paracrine effects and thereby change the microenvironment in the deeper layers. Consequently, distant cells may benefit from cell-cell communication via paracrine mechanisms. Furthermore, there are always encounters with immune system cells, which are able to move across tissues and evoke a response at distant sites (e.g. immunogenic cell death) [39].

Taken together, these biological activities can explain the photorejuvenative effect of CAP that was previously demonstrated clinically [40]. In addition, the improvement of dermal photodamage could also theoretically reduce the pathogenetic role of damaged dermis in the development of AKs [41].

This study has a number of limitations. First, and most importantly, the number of patients enrolled in the study was relatively small, which may have affected the statistical power of the observed results. Secondly, response to treatment was only clinically evaluated, without an extensive and in-depth histological confirmation (performed in only two patients) that would have corroborated our findings. This methodical choice was necessary due to the difficulties of recruiting patients suitable for plasma therapy and the ethical implications of performing skin biopsies of purely experimental significance on these patients in aesthetic areas of the face. Finally, we did not compare our results with those resulting from the application of other physical field therapies, such as photodynamic therapy.

\section{CONCLUSION}

In conclusion, the present investigation has demonstrated that CAP treatment is effective for reducing the cumulative AK area, the AKASI score and the number of AKs. It is our belief that digital assessment of the reduction in the cumulative AK area (preceded by a careful inspection of the treated area with digital dermoscopy) should be the performance index of choice for the evaluation of the efficacy of field treatments for AKs. In addition, we have demonstrated that CAP treatment was not followed by skin atrophy and that it improved HFUS features of chronic photodamage of the dermis of the skin underlying and surrounding the AK spots.

Future results on larger case series will enable us to compare the efficacy of different physical field treatments and to better outline the changes to skin cells induced by CAP treatment.

\section{ACKNOWLEDGEMENTS}

The authors thank the participants of the study with a special appreciation to the nursing staff of the phototherapy and laser unit. The corresponding author thanks nurse Nicoletta Barbera for her highly valued collaboration.

Funding. No funding or sponsorship was received for this study or publication of this article.

Authorship. All named authors meet the International Committee od Medical Journal Editors (ICMJE) criteria for authorship for this article, take responsibility for the integrity of the work as a whole, and have given their approval for this version to be published.

Disclosures. Mariachiara Arisi, Simone Soglia, Edoardo Guasco Pisani, Anna Venturuzzo, Alessandra Gelmetti, Cesare Tomasi, Cristina Zane, Mariateresa Rossi and Luisa Lorenzi have nothing to disclose. Piergiacomo Calzavara-Pinton is a member of the journal's Editorial Board. 
Compliance with Ethics Guidelines. The study was approved by the Local Ethics Committee (Protocol Number 4195) and was conducted in accordance with the Declaration of Helsinki. All patients were given verbal and written information on the nature of the study, and all signed an informed consent before enrolment. All patients provided written informed consent for the publication of their cases and images.

Data Availability. Due to local ethic limitations, study data are not deposited in a public repository.

Open Access. This article is licensed under a Creative Commons Attribution-NonCommercial 4.0 International License, which permits any non-commercial use, sharing, adaptation, distribution and reproduction in any medium or format, as long as you give appropriate credit to the original author(s) and the source, provide a link to the Creative Commons licence, and indicate if changes were made. The images or other third party material in this article are included in the article's Creative Commons licence, unless indicated otherwise in a credit line to the material. If material is not included in the article's Creative Commons licence and your intended use is not permitted by statutory regulation or exceeds the permitted use, you will need to obtain permission directly from the copyright holder. To view a copy of this licence, visit http://creativecommons.org/licenses/by$\mathrm{nc} / 4.0 /$.

\section{REFERENCES}

1. Semmler ML, Bekeschus S, Schäfer M, et al. Molecular mechanisms of the efficacy of cold atmospheric pressure plasma (CAP) in cancer treatment. Cancers (Basel). 2020;12:269.

2. Kim JY, Wei Y, Li J, Kim SO. 15- $\mu$ m-sized singlecellular-level and cell-manipulatable microplasma jet in cancer therapies. Biosens Bioelectron. 2010;26:555-9.

3. Babington P, Rajjoub K, Canady J, Siu A, Keidar M, Sherman JH. Use of cold atmospheric plasma in the treatment of cancer. Biointerphases. 2015;10: 029403.

4. Raiser J, Zenker M. Argon plasma coagulation for open surgical and endoscopic applications: state of the art. J Phys D Appl Phys. 2006;39:3520-3.

5. Fridman G, Friedman G, Gutsol A, Shekhter AB, Vasilets VN, Fridman A. Applied plasma medicine. Plasma Process Polym. 2008;5:503-33.

6. Kalghatgi S, Friedman G, Fridman A, Clyne AM. Endothelial cell proliferation is enhanced by low dose non-thermal plasma through fibroblast growth factor-2 release. Ann Biomed Eng. 2010;38: 748-57.

7. Laroussi M. Low temperature plasma-based sterilization: overview and state-of-the-art. Plasma Process Polym. 2005;2:391-400.

8. Kim $\mathrm{CH}$, Bahn $\mathrm{JH}$, Lee $\mathrm{SH}$, et al. Induction of cell growth arrest by atmospheric non-thermal plasma in colorectal cancer cells. J Biotechnol. 2010;150: 530-8.

9. Bernhardt T, Semmler ML, Schäfer M, Bekeschus S, Emmert S, Boeckmann L. Plasma medicine: applications of cold atmospheric pressure plasma in dermatology. Oxid Med Cell Longev. 2019;3(2019): 3873928 .

10. Dubuc A, Monsarrat P, Virard F, et al. Use of coldatmospheric plasma in oncology: a concise systematic review. Ther Adv Med Oncol. 2018;10: 1758835918786475.

11. Keidar M, Walk R, Shashurin A, et al. Cold plasma selectivity and the possibility of a paradigm shift in cancer therapy. Br J Cancer. 2011;105:1295-301.

12. Mashayekh AS, Rajaee H, Akhlaghi M, Shokri B, Hassan Z. Atmospheric-pressure plasma jet characterization and applications on melanoma cancer treatment (B/16-F10). Phys Plasma. 2015; 22: 093508. https://doi.org/10.1063/1.4930536.

13. Daeschlein G, Scholz S, Lutze S, et al. Comparison between cold plasma, electrochemotherapy and combined therapy in a melanoma mouse model. Exp Dermatol. 2013;22:582-6.

14. Metelmann HR, Seebauer C, Miller V, et al. Clinical experience with cold plasma in the treatment of locally advanced head and neck cancer. Clin Plasma Med. 2018;9:6-13.

15. Metelmann HR, Nedrelow DS, Seebauer C, et al. Head and neck cancer treatment and physical plasma. Clin Plasma Med. 2015;3:17-23. 
16. Friedman PC, Miller V, Fridman G, Lin A, Fridman A. Successful treatment of actinic keratoses using nonthermal atmospheric pressure plasma: a case series. J Am Acad Dermatol. 2017;76:349-50.

17. Wirtz M, Stoffels I, Dissemond J, Schadendorf D, Roesch A. Actinic keratoses treated with cold atmospheric plasma. J Eur Acad Dermatol Venereol. 2018;32:e37-9.

18. Yan D, Sherman JH, Keidar M. Cold atmospheric plasma, a novel promising anti-cancer treatment modality. Oncotarget. 2017;8:15977-95.

19. Ma Y, Ha CS, Hwang SW, et al. Non-thermal atmospheric pressure plasma preferentially induces apoptosis in p53-mutated cancer cells by activating ROS stress-response pathways. PLoS ONE. 2014;9: e91947.

20. Keidar M, Shashurin A, Volotskova O, et al. Cold atmospheric plasma in cancer therapy. Phys Plasma. 2013;20:057101.

21. Fernandez Figueras MT. From actinic keratosis to squamous cell carcinoma: pathophysiology revisited. J Eur Acad Dermatol Venereol. 2017;31:5-7.

22. Heppt MV, Leiter U, Steeb T, et al. S3 guideline for actinic keratosis and cutaneous squamous cell carcinoma: short version, part 1: diagnosis, interventions for actinic keratoses, care structures and quality-of-care indicators. J Dtsch Dermatol Ges. 2020;18:275-94.

23. Friedman PC, Miller V, Fridman G, Fridman A. Various cold plasma devices for the treatment of actinic keratosis. J Eur Acad Dermatol Venereol. 2018;32:e445-6.

24. Olsen EA, Abernethy ML, Kulp-Shorten C, et al. A double-blind, vehicle-controlled study evaluating masoprocol cream in the treatment of actinic keratoses on the head and neck. J Am Acad Dermatol. 1991;24:738-43.

25. Hemkle A, Gerling T, Weltmann KD (2018). Plasma sources for biomedical applications. In: Metelmann HR, von Woedtke T, Weltmann KD, editors. Comprehensive clinical plasma medicine. Cham: Springer. p. 23-41.

26. Calzavara-Pinton $\mathrm{P}$, Zane $\mathrm{C}$, Arisi M, Hamon PA, Tanova NT. Evaluation of the costs of topical treatments for actinic keratosis based on lesion response and the affected area. G Ital Dermatol Venereol. 2018;153:764-75.

27. Arisi M, Zane C, Polonioli M, et al. Effects of MALPDT, ingenol mebutate and diclofenac plus hyaluronate gel monitored by high-frequency ultrasound and digital dermoscopy in actinic keratosis- a randomized trial. J Eur Acad Dermatol Venereol. 2020;34:1225-32.

28. Dirschka T, Pellacani G, Micali G, et al. A proposed scoring system for assessing the severity of actinic keratosis on the head: actinic keratosis area and severity index. J Eur Acad Dermatol Venereol. 2017;31:1295-302.

29. Babilas P, Kohl E, Maisch T, et al. In vitro and in vivo comparison of two different light sources for topical photodynamic therapy. Br J Dermatol. 2006;154:712-8.

30. Dirschka T, Gupta G, Micali G, et al. Real-world approach to actinic keratosis management: practical treatment algorithm for office-based dermatology. J Dermatolog Treat. 2017;28:431-42.

31. Calzavara-Pinton P, Hædersdal M, Barber K, et al. Structured expert consensus on actinic keratosis: treatment algorithm focusing on daylight PDT. J Cutan Med Surg. 2017;21:3S-16S.

32. Schmitz L, Hessam S, Scholl L, et al. Argon plasma coagulation of actinic keratoses imaged by optical coherence tomography: an in vivo study indicating a possible lesion-directed treatment. J Biophotonics. 2018;11:e201800075.

33. Fernández-Figueras MT, Carrato C, Sáenz X, et al. Actinic keratosis with atypical basal cells (AK I) is the most common lesion associated with invasive squamous cell carcinoma of the skin. J Eur Acad Dermatol Venereol. 2015;29:991-7.

34. Sandby-Møller J, Wulf HC. Ultrasonographic subepidermal low-echogenic band, dependence of age and body site. Skin Res Technol. 2004;10:57-63.

35. Arndt S, Schmidt A, Karrer S, von Woedtke T. Comparing two different plasma devices kINPen and Adtec SteriPlas regarding their molecular and cellular effects on wound healing. Clin Plasma Med. 2018;9:24-33.

36. Gan L, Zhang S, Poorun D, et al. Medical applications of nonthermal atmospheric pressure plasma in dermatology. J Dtsch Dermatol Ges. 2018;16: 7-13.

37. Arndt S, Unger $\mathrm{P}$, Wacker E, et al. Cold atmospheric plasma (CAP) changes gene expression of key molecules of the wound healing machinery and improves wound healing in vitro and in vivo. PLoS ONE. 2013;8:e79325.

38. Penn JW, Grobbelaar AO, Rolfe KJ. The role of the TGF- $\beta$ family in wound healing, burns and scarring: a review. Int J Burns Trauma. 2012;2:18-28. 
39. Privat-Maldonado A, Schmidt A, Lin A, et al. ROS from physical plasmas: redox chemistry for biomedical therapy. Oxid Med Cell Longev. 2019;2019:9062098.

40. Heinlin J, Isbary G, Stolz W, et al. Plasma applications in medicine with a special focus on dermatology. J Eur Acad Dermatol Venereol. 2011;25:1-11.

41. Procopio MG, Laszlo C, Al Labban D, et al. Combined CSL and p53 downregulation promotes cancer-associated fibroblast activation. Nat Cell Biol. 2015;17:1193-204. 